\title{
Ligand Control of Room-Temperature Phosphorescence Violating Kasha's Rule in Hybrid Organic-Inorganic Metal Halides
}

Li-Li Zhu, ${ }^{a}$ Yue-E Huang, ${ }^{a}$ Liao-Kuo Gong, ${ }^{b}$ Xiao-Ying Huang, ${ }^{b}$ Xing-Hui Qi, ${ }^{b}$ Xiao-Hui Wu, ${ }^{* a}$ and KeZhao Du*a

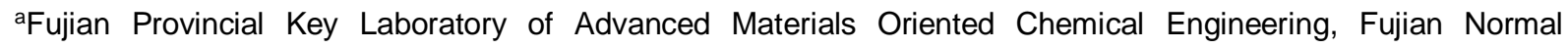
University, 32 Shangsan Road, Fuzhou 350007, China

bState Key Laboratory of Structural Chemistry, Fujian Institute of Research on the Structure of Matter, Chinese Academy of Sciences, Fuzhou, Fujian 350002, People's Republic of China

\section{Experimental synthesis}

\section{Reagents}

Lead(II) bromide $\left(\mathrm{PbBr}_{2}, 99 \%\right.$, Adamas), terephthalic acid $\left(\mathrm{HO}_{2} \mathrm{C}\left(\mathrm{C}_{6} \mathrm{H}_{4}\right) \mathrm{CO}_{2} \mathrm{H}, 99 \%\right.$, Adamas), sodium hydroxide $(\mathrm{NaOH}, \geq 96 \%$, Greagent), 1,4-Phenylenediacetic acid $\left(\mathrm{HO}_{2} \mathrm{C}_{2}\left(\mathrm{C}_{6} \mathrm{H}_{4}\right) \mathrm{C}_{2} \mathrm{O}_{2} \mathrm{H}, 98 \%+\right.$ Adamas $)$, Lead(II) Nitrate $\left(\mathrm{Pb}\left(\mathrm{NO}_{3}\right)_{2}, \quad\right.$ AR, Sinopharm Chemical ReagentCo., Ltd) and N,N-Dimethylformamide $\left(\mathrm{C}_{3} \mathrm{H}_{7} \mathrm{NO}\right.$, aladdin, $\left.\geqslant 99.5 \%\right)$ were used without further purification.

\section{Synthesis}

$\left[\mathrm{Pb}_{4} \mathrm{Br}_{2}\right]\left[\mathrm{O}_{2} \mathrm{C}(\mathrm{CH})_{6} \mathrm{CO}_{2}\right]_{3}(\mathrm{TPAPbBr}) .0 .031 \mathrm{~g}$ Terephthalic Acid (TPA) (0.1875 mmol) and $0.015 \mathrm{~g} \mathrm{NaOH}(0.375 \mathrm{mmol})$ was put into a $20 \mathrm{ml}$ Teflon-lined autoclave reactor with $2 \mathrm{ml}$ pure water, and then gave a stirred condition about 5 minutes until obtain a clear liquid. 0.092 g $\operatorname{PbBr}_{2}(0.25 \mathrm{mmol})$ were added into the precursor solution and then stirred 15 minutes until the mixture was thoroughly dispersed. Subsequently, the autoclave was enclosed in a stainless steel tank and heated at $140{ }^{\circ} \mathrm{C}$ for $56 \mathrm{~h}$, and then the reaction system was cooled slowly to 
room temperature to obtain the mixed products. The products were washed three times with dimethylformamide (DMF) to get colorless bulk TPAPbBr crystals.

$\mathrm{Pb}_{2}(\mathrm{PLA})\left(\mathrm{H}_{2} \mathrm{O}\right)_{2} \mathrm{Br}_{2}(\mathrm{PLAPbBr}) . \quad$ A mixture of 0.0243 g 1,4-Phenylenediacetic acid (PLA) $(0.125 \mathrm{mmol}), 0.01 \mathrm{~g} \mathrm{NaOH}(0.25 \mathrm{mmol})$ and $3 \mathrm{ml}$ pure water was put into a $20 \mathrm{ml}$ Teflonlined autoclave reactor with stirring until the solid was dissolved into a transparent pale yellow liquid. $0.092 \mathrm{~g} \mathrm{PbBr}_{2}(0.25 \mathrm{mmol})$ was dispersed into the precursor thoroughly. Subsequently, the autoclave was enclosed in a stainless steel tank and heated at $140{ }^{\circ} \mathrm{C}$ for 72 $\mathrm{h}$, and then the mixed products were obtained when the reaction system was cooled slowly to the room temperature. After washed three times with DMF, the yellowish sheet PLAPbBr crystals were selected out for the further characterization.

$\boldsymbol{P b}\left[\mathrm{O}_{2} \mathrm{C}(\mathrm{CH})_{6} \mathrm{CO}_{2}\right](\mathrm{TPAPb}) . \quad 0.0415 \mathrm{~g}$ TPA $(0.25 \mathrm{mmol})$ was weighted in a $20 \mathrm{ml}$ Teflonlined autoclave reactor and dissolved into $2 \mathrm{ml} \mathrm{DMF}$. $0.083 \mathrm{~g} \mathrm{~Pb}\left(\mathrm{NO}_{3}\right)_{2}(0.25 \mathrm{mmol})$ were added into the precursor solution. Subsequently, the autoclave was enclosed in a stainless steel tank and heated at $120{ }^{\circ} \mathrm{C}$ for $9 \mathrm{~h}$. And then the reaction system was cooled slowly to the room temperature to obtain colorless bulk TPAPb crystals.

$\mathrm{Pb}\left[\mathrm{O}_{2} \mathrm{C}_{2}(\mathrm{CH})_{6} \mathrm{C}_{2} \mathrm{O}_{2}\right](\mathrm{PLAPb})$. Replacing TPA with PLA in the synthesis procedure of $\mathrm{TPAPb}$, yellow rice-granule PLAPb crystals were obtained.

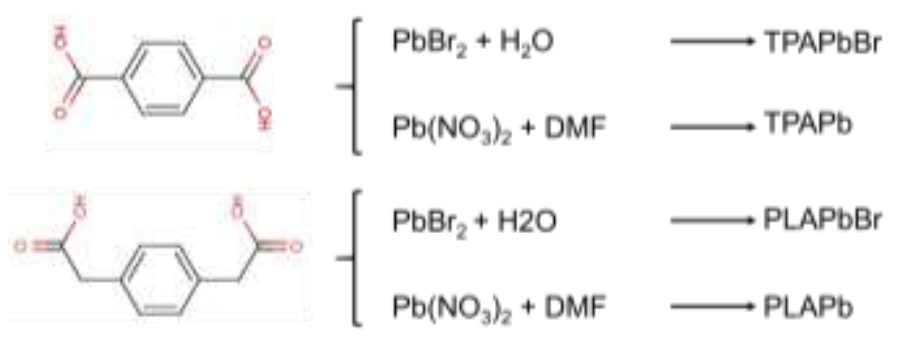

Scheme 1 . The synthesis routines for the four compounds 


\section{Characterization methods}

Single Crystal X-ray diffraction (SC-XRD). Suitable single crystals of TPAPbBr, TPAPb, $\mathrm{PLAPbBr}$ and $\mathrm{PLAPb}$ were selected by hand and then glued on the top of thin glass fibers, respectively. The single-crystal X-ray diffraction data was tested on a SuperNova CCD diffractometer with $\mathrm{MoK} \alpha$ radiation $(\lambda=0.71073 \AA)$ at $293 \mathrm{~K}$ or $150 \mathrm{~K}$. The structures were solved via direct methods and refined via full-matrix least-squares on F2 using Shelxl and Olex software. ${ }^{[1,2]}$ All atoms were refined anisotropically except hydrogen.

Powder X-ray diffraction (PXRD). The PXRD was collected via Rigaku Miniflex- II diffractometer with $\mathrm{Cu} K \alpha$ radiation $(\lambda=1.54178 \AA)$ in the angular range of $2 \theta=5-65^{\circ}$ at room temperature with a scan speed of $1 \%$ min. The simulated PXRD was calculated via Mercury software based on the corresponding single-crystal data.

Thermogravimetry analysis (TGA). TGA was proceeded on NETZSCH STA 449F3 with a heating rate of $10 \mathrm{~K} \cdot \mathrm{min}^{-1}$ under a dry $\mathrm{N}_{2}$ atmosphere.

Optical properties. PL (photoluminescence), PLE (photoluminescence excitation) and delayed-PL were collected on PerkinElmer LS55 fluorescence spectrometer. The temperature dependent TRPL (time-resolved photoluminescence) and low temperature PL were collected on Edinburgh FL980 UV/V/NIR fluorescence spectrometer. Quantum yields (QYs) were acquired via Edinburgh FLS 1000 UV/V/NIR fluorescence spectrometer.

Elemental analysis (EA) EA for C, H, N were measured via using German Elementary Vario EL III equipment 
(a)

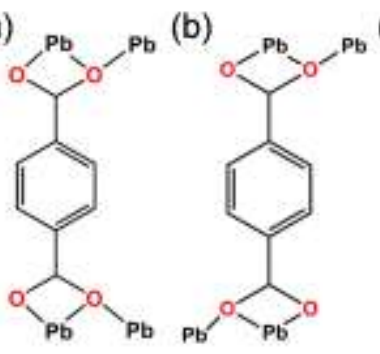

(c)<smiles>COC(O[PH3])c1ccc(C2OPO2)cc1</smiles>

d)

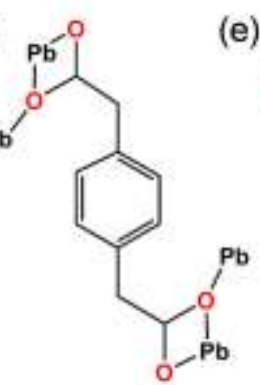

(e)

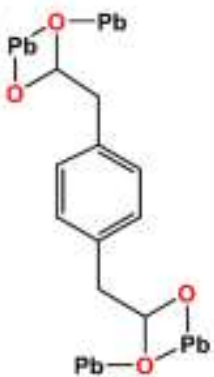

Figure S1. The coordination modes for the conjugate carboxylic acid in $\operatorname{TPAPbBr}(a, b)$, $\mathrm{TPAPb}(\mathrm{c}), \mathrm{PLAPbBr}(\mathrm{d})$ and PLAPb (e)

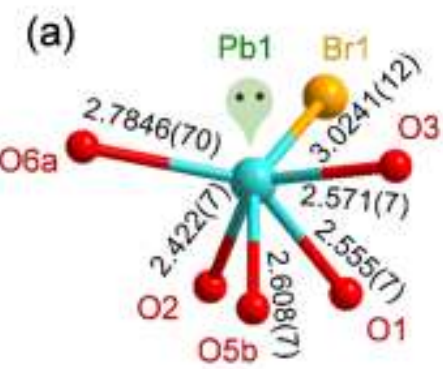

(d) (b)

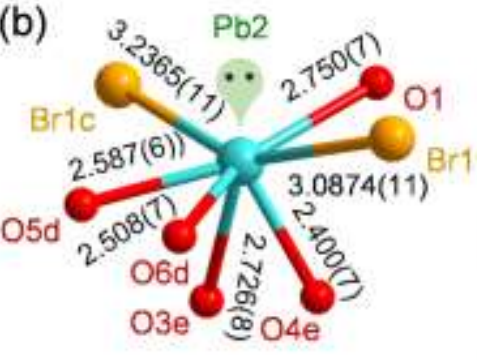

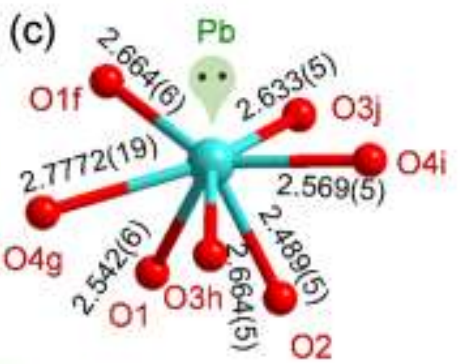
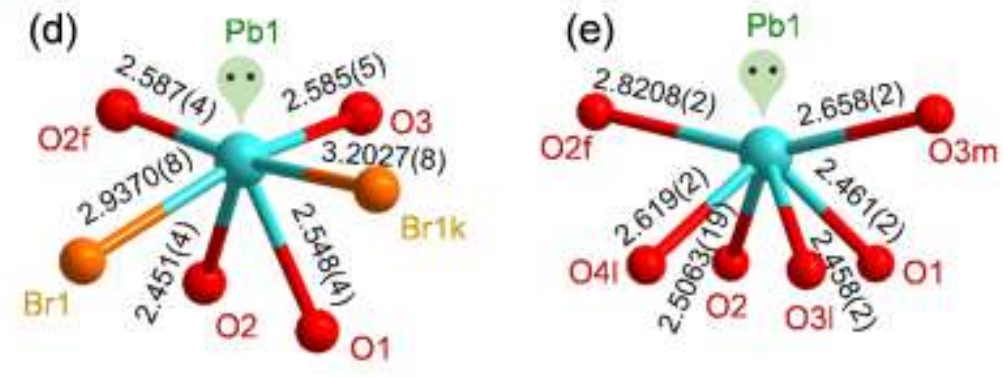

Figure S2. The coordination modes for the $\mathrm{Pb}$ atoms in $\operatorname{TPAPbBr}(\mathrm{a}, \mathrm{b}), \operatorname{TPAPb}(\mathrm{c})$, $\operatorname{PLAPbBr}(\mathrm{d})$ and PLAPb (e). The symmetry operations: (a) $-1+x, 0.5-y, 0.5+z$; (b) $x, 0.5-$ $y, 0.5+z ;$ (c) $1+x, y, z$; (d) $1-x,-y,-z ;$ (e) $1-x,-0.5+y, 0.5-z$; (f) $1-x, 1-y, 1-z ;$ (g) 0.5 $+x, y, 1.5-z$; (h) $1-x,-0.5+y, 1.5-z$; (i) $x, 0.5-y,-0.5+z$; (j) $0.5-\mathrm{x}, 1-y,-0.5+z$; (k) $2-$ $z, 2-y, 1-z ;(\mathrm{l}) 1+x, 1.5-y,-0.5+z ;(\mathrm{m}) 1-x,-0.5+y, 1.5-z$. 

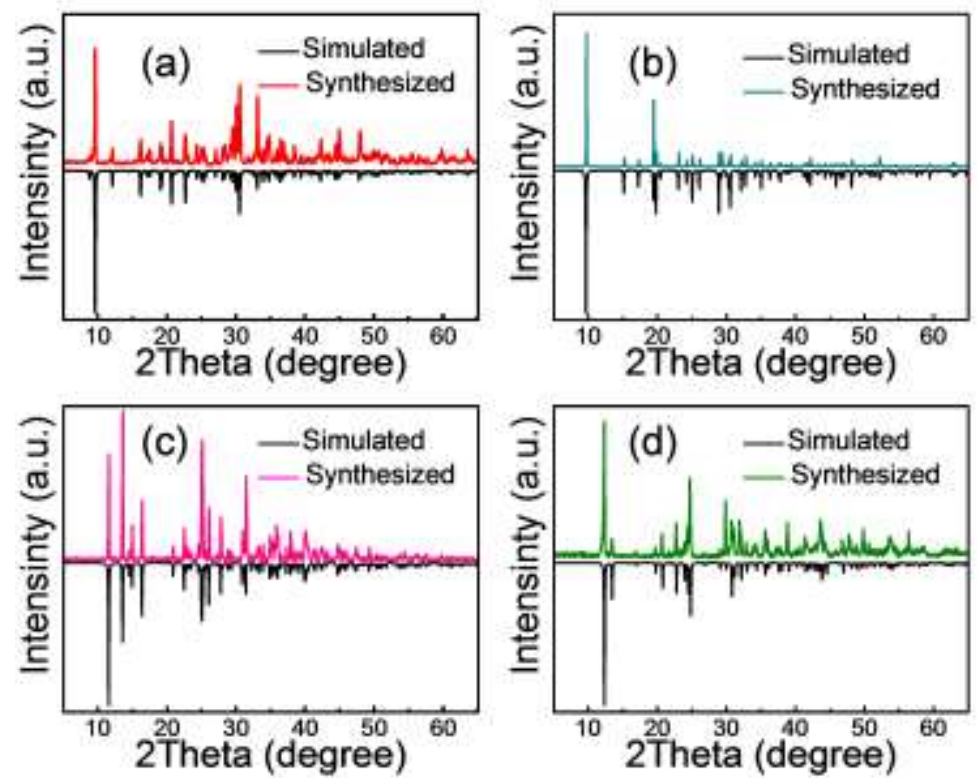

Figure S3. The PXRD of TPAPbBr (a), TPAPb (b), PLAPbBr (c) and PLAPb (d).
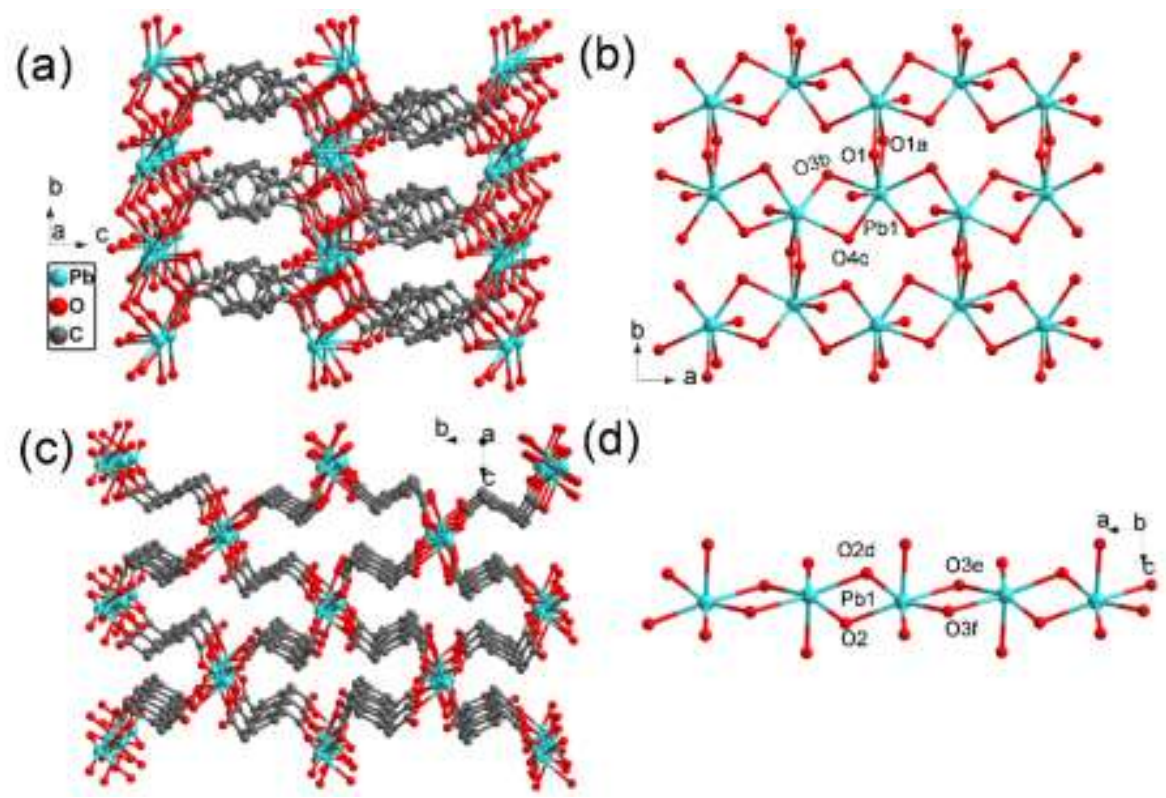

Figure S4. The 3D stacked diagrams of TPAPb (a) and PLAPb (c). Crystal structure detail of $\left[\mathrm{PbO}_{4}\right]$ layer of TPAPb (b) and $\left[\mathrm{PbO}_{4}\right]$ chain of PLAPb (d). The symmetry operations: (a) 1 $x, 1-y, 1-z$; (b) $0.5-x, 1-y,-0.5+z$; (c) $x, 0.5-y,-0.5+z$; (d) $1-x, 1-y, 1-z$; (e) $1+x, 1.5-y$, $-0.5+z ;$ (f) $1-x,-0.5+y, 1.5-z$. 
$\mathrm{TPAPb}$ is crystallized in orthorhombic $\mathrm{Pbca}$ space group. There is one independent $\mathrm{Pb}$ atoms and one TPA molecule in each asymmetric unit. $\mathrm{Pb}$ adopts a hemi-directed sphere coordinated by seven $\mathrm{O}$ atoms. The $\mathrm{PbO}_{7}$ unit interconnects with each other to form a $\mathrm{PbO}_{5}$ chain by sharing $\mathrm{O} 3 \mathrm{~b}-\mathrm{O} 4 \mathrm{c}$ edge. Then the $\mathrm{PbO}_{5}$ chain are extended into a layered $\mathrm{PbO}_{4}$ by sharing O1-O1a edge. All the $\mathrm{O}$ atoms mentioned above are from the TPA ligand, which connect with the $\mathrm{PbO}_{4}$ layer into a three-dimensional framework (Figure $\mathrm{S} 4 \mathrm{a}$ and $4 \mathrm{~b}$ ).

$\mathrm{PLAPb}$ belongs to the monoclinic $P 2{ }_{1} / c$ space group. There is one independent $\mathrm{Pb}$ atom and one PLA molecule in each asymmetric unit. $\mathrm{Pb}$ adopts a hemi-directed sphere coordinated by six $\mathrm{O}$ atoms. The neighboring $\mathrm{Pb}$ atoms are edge-shared by $\mathrm{O} 3 \mathrm{e}-\mathrm{O} 3 \mathrm{f}$ and $\mathrm{O} 2-\mathrm{O} 2 \mathrm{~d}$ to generate $\mathrm{abO}_{4}$ chain, which is further assembled by PLA molecules leading to the threedimensional PLAPb (Figure S4c and 4d).
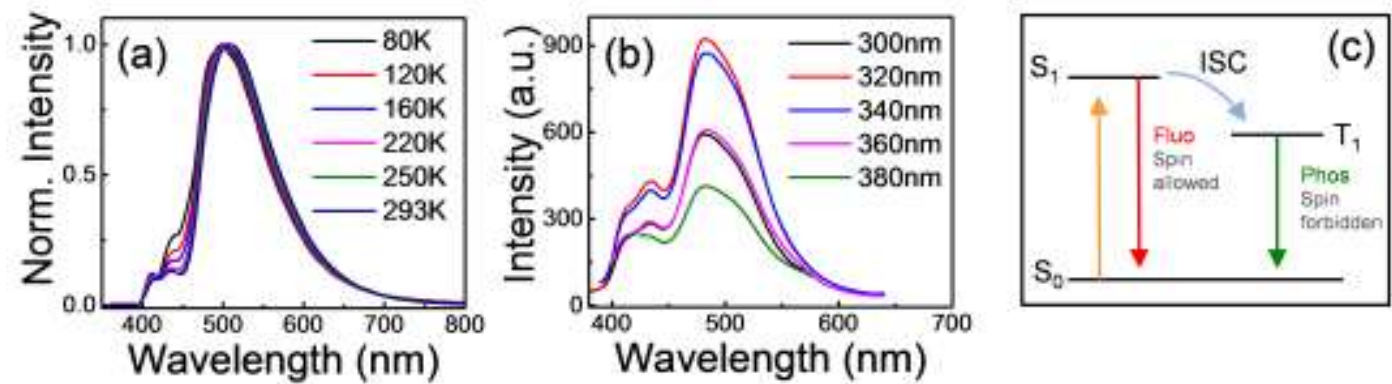

Figure S5. The temperature-dependent $\left(\lambda_{\mathrm{ex}}=330 \mathrm{~nm}\right)(\mathrm{a})$ and excitation-dependent (b) PL spectra of $\mathrm{TPAPbBr}$; (c) The schematic diagram of fluorescence and phosphorescence generation mechanism. $S_{0}=$ ground state; $S_{1}=$ the lowest excited singlet state; $T_{1}=$ the lowest triplet state; ISC $=$ intersystem crossing. 

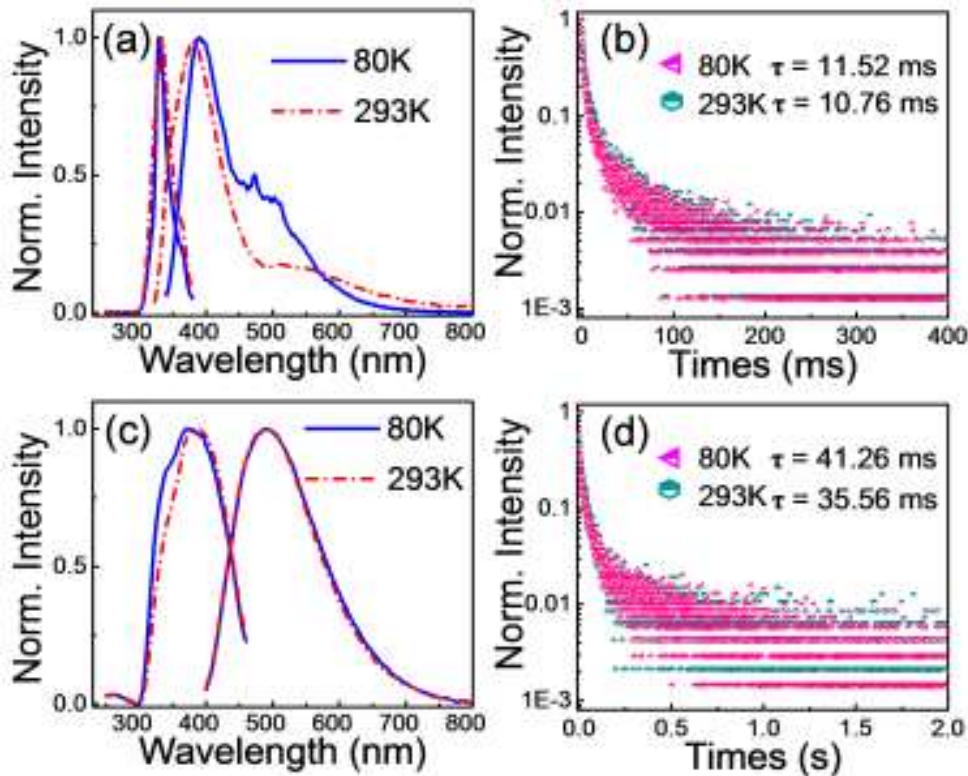

Figure S6. The PLE and PL spectra of TPA $\left(\lambda_{\mathrm{ex}}=330 \mathrm{~nm}, \lambda_{\mathrm{em}}=380 \mathrm{~nm}\right)(\mathrm{a})$ and PLA $\left(\lambda_{\mathrm{ex}}=\right.$ $390 \mathrm{~nm}, \lambda_{\mathrm{em}}=480 \mathrm{~nm}$ ) (c) with different temperatures; The TRPL of TPA (b) and PLA (d) at $380 \mathrm{~nm}$ and 480nm, respectively, under different temperatures.
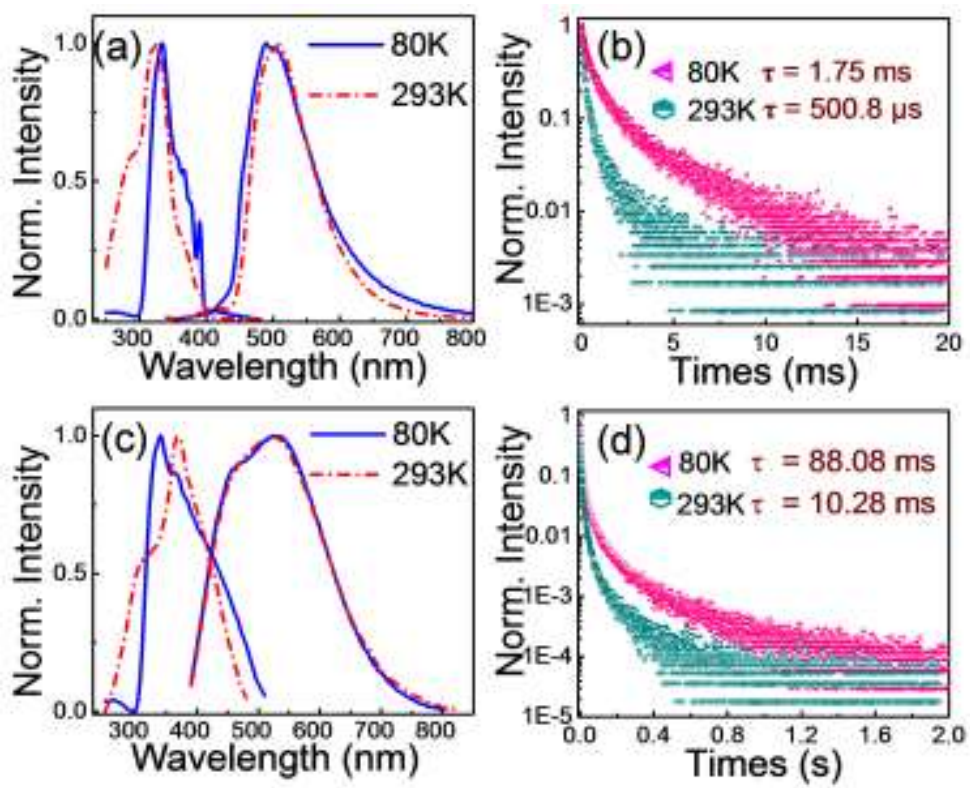
Figure S7. The PLE and PL spectra of TPAPb $\left(\lambda_{\mathrm{ex}}=330 \mathrm{~nm}, \lambda_{\mathrm{em}}=500 \mathrm{~nm}\right)(\mathrm{a})$ and PLAPb $\left(\lambda_{\mathrm{ex}}=370 \mathrm{~nm},\left(\lambda_{\mathrm{em}}=530 \mathrm{~nm}\right)(\mathrm{c})\right.$ with different temperatures; The TRPL of TPAPb $(\mathrm{b})$ and $\mathrm{PLAPb}(\mathrm{d})$ at 500nm and 530nm, respectively, at different temperatures.
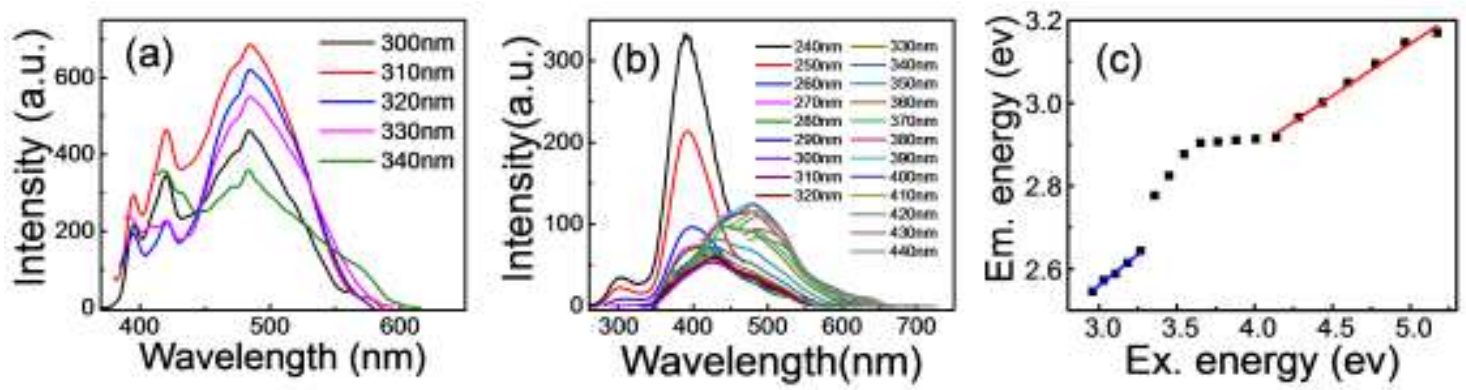

Figure S8. The excitation-dependent PL spectra of TPAPb (a), PLAPb (b); (c) The emission peak energy of PLAPb dependent on the corresponding excitation energy (Ex. = excitation; Em. $=$ emission; The red line: $\mathrm{Y}=1.891+0.250 \mathrm{X}, \mathrm{R} 2=0.986$; The bule line: $Y=1.648+$ $\left.0.304 X, R^{2}=0.992\right)$
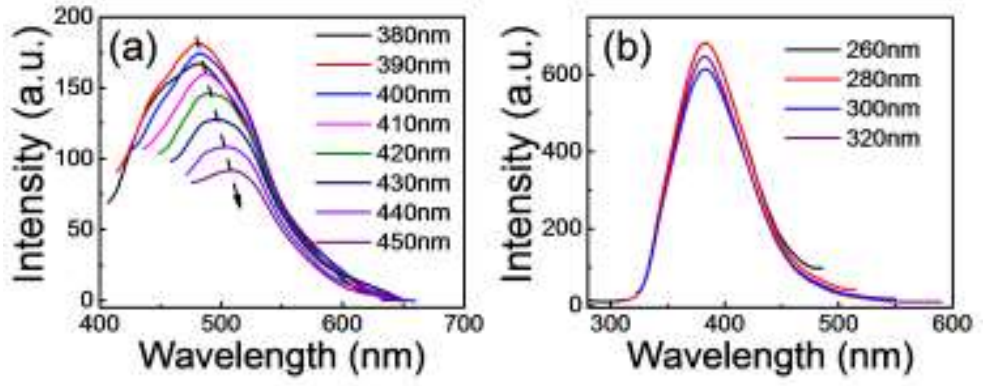

Figure S9. The excitation-dependent PL spectra of PLA (a) and TPA (b). 


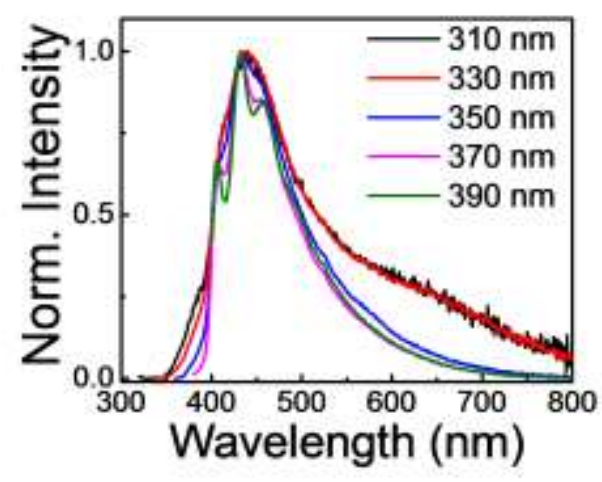

Figure S10. The excitation-dependent PL spectra of PLAPbBr at $80 \mathrm{~K}$.

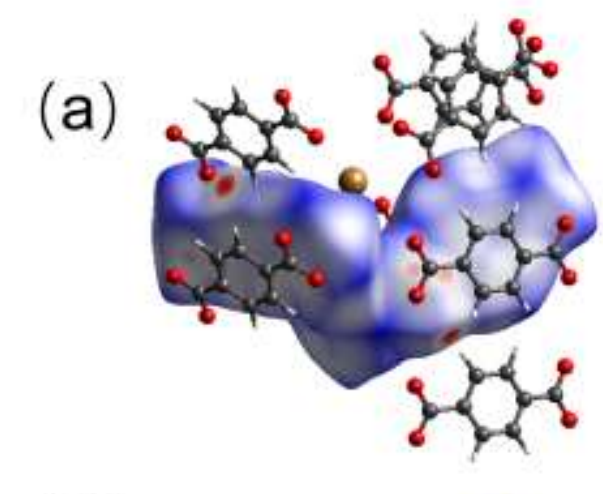

(b)
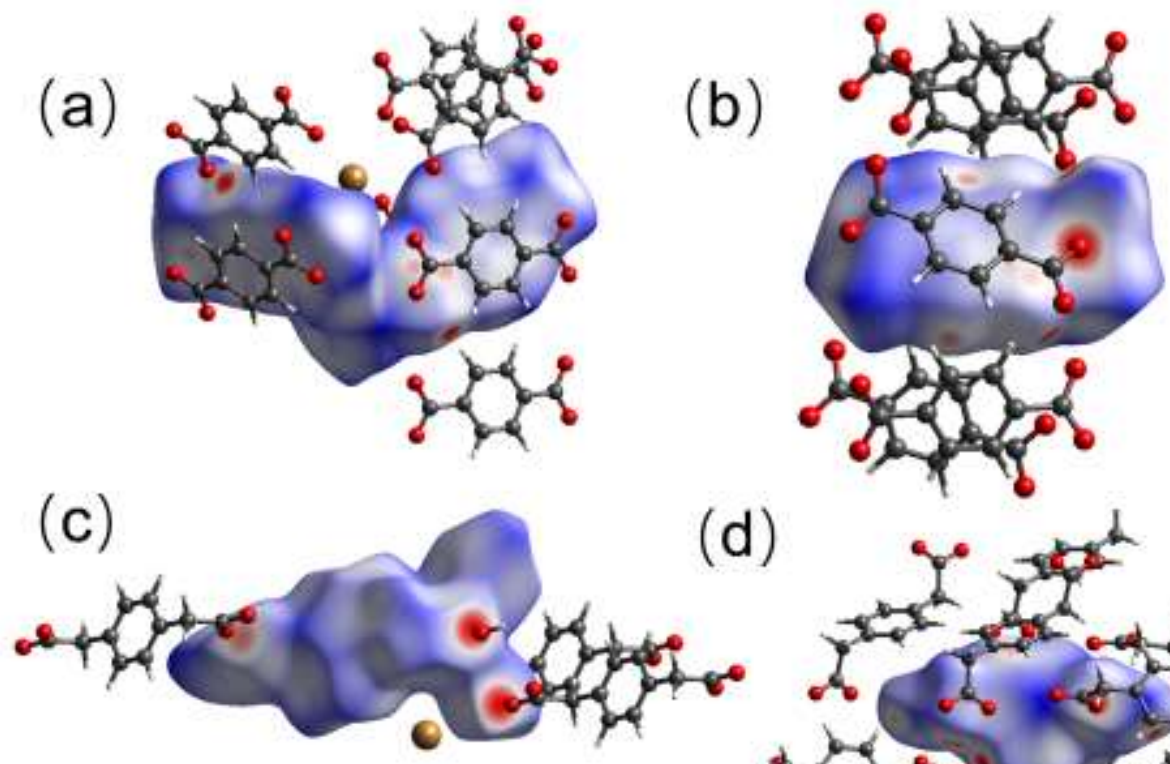

(d)

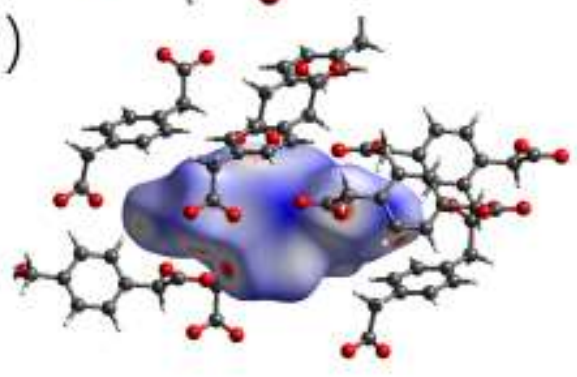

Figure S11. Hirshfeld surface ( $d_{\text {norm}}$ ) analysis of the organic ligands in TPAPbBr $(a), \operatorname{TPAPb}$ (b), PLAPbBr (c) and PLAPb (d). 

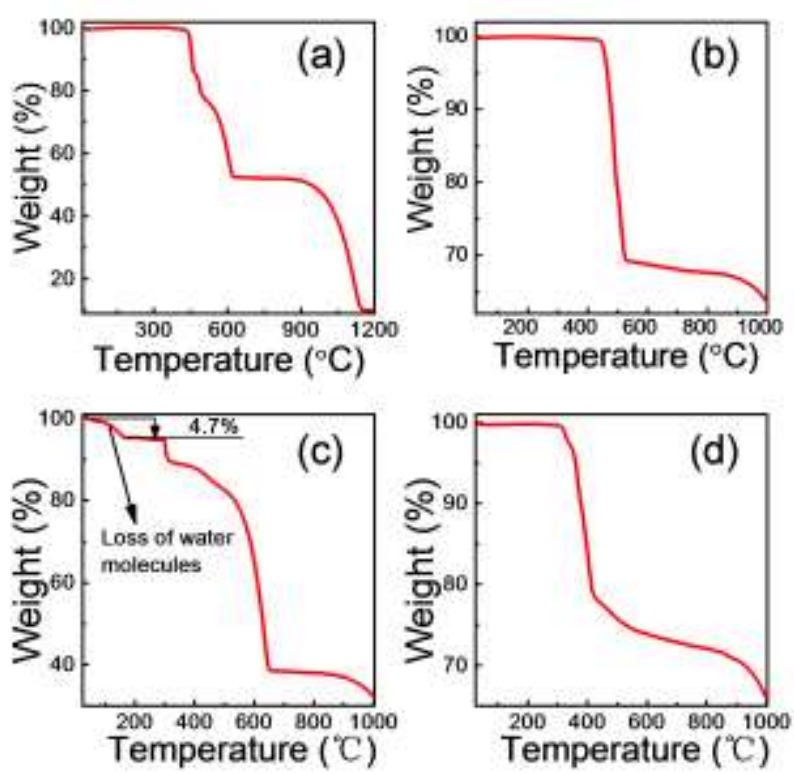

Figure S12. Thermogravimetric analysis curve of TPAPbBr (a), TPAPb (b), PLAPbBr (c) and PLAPb (d).

Table S1. The elemental analysis for TPAPbBr and PLAPbBr.

\begin{tabular}{|c|c|c|c|c|c|c|}
\hline & \multicolumn{3}{|l|}{ TPAPbBr } & \multicolumn{3}{|l|}{ PLAPbBr } \\
\hline & $\mathrm{C}$ & $\mathrm{H}$ & $\mathrm{N}$ & $\mathrm{C}$ & $\mathrm{H}$ & $\mathrm{N}$ \\
\hline Calculated (\%) & 19.45 & 0.81 & 0 & 14.96 & 1.49 & 0 \\
\hline Found (\%) & $18.72-18.75$ & $0.85-0.79$ & $=<0.3$ & $15.18-15.19$ & $1.43-1.47$ & $=<0.3$ \\
\hline
\end{tabular}

Table S2. Summary of the crystal data and structure refinements for TPAPbBr and TPAPb.

\begin{tabular}{lll}
\hline Compound name & TPAPbBr & TPAPb \\
\hline Empirical formula & $\mathrm{C}_{12} \mathrm{H}_{6} \mathrm{BrO}_{6} \mathrm{~Pb}_{2}$ & $\mathrm{C}_{8} \mathrm{H}_{4} \mathrm{O}_{4} \mathrm{~Pb}$ \\
Formula weight & 740.46 & 371.30 \\
Crystal size $\left(\mathrm{mm}^{3}\right)$ & $0.3 \times 0.2 \times 0.2$ & $0.3 \times 0.2 \times 0.2$ \\
Crystal system & Monoclinic & orthorhombic \\
Space group & $P 2_{1} / c$ & $P b c a$ \\
$T / \mathrm{K}$ & $298.25(10)$ & $293(2)$ \\
$\lambda / \AA$ & 0.71073 & 0.71073 \\
\hline
\end{tabular}




\begin{tabular}{|c|c|c|}
\hline$a / \AA$ & $6.1105(2)$ & $7.716(7)$ \\
\hline$b / \AA$ & $12.0397(5)$ & $10.285(8)$ \\
\hline$c / \AA$ & $18.4209(5)$ & $18.280(15)$ \\
\hline$\alpha /{ }^{\circ}$ & 90 & 90 \\
\hline$\beta /^{\circ}$ & $91.547(3)$ & 90 \\
\hline$\gamma /{ }^{\circ}$ & 90 & 90 \\
\hline$V / \AA^{3}$ & $1354.72(8)$ & 1451(2) \\
\hline $\mathrm{Z}$ & 4 & 8 \\
\hline$D_{c} / \mathrm{g} \cdot \mathrm{cm}^{-3}$ & 3.630 & 3.400 \\
\hline$\mu / \mathrm{mm}^{-1}$ & 27.795 & 23.232 \\
\hline$F(000)$ & 1300.0 & 1328.0 \\
\hline $2 \theta$ for data collection $\left(^{\circ}\right)$ & $7.122-53.826$ & $6.912-54.958$ \\
\hline Measured refls. & 8717 & 10571 \\
\hline Independent refls. & 2907 & 1653 \\
\hline Observed reflection $(I>2 \sigma(I)$ & 2468 & 1935 \\
\hline Peak and hole/ e $\AA^{-3}$ & $1.26 /-1.94$ & $2.84 /-2.86$ \\
\hline$R_{\text {int }}$ & 0.0564 & 0.1141 \\
\hline No. of parameters & 190 & 118 \\
\hline GOF & 1.019 & 0.989 \\
\hline${ }^{\mathrm{a}} R_{1}, \mathrm{w} R_{2}[I>2 \sigma(I)]$ & $0.0382,0.0710$ & $0.0414,0.1037$ \\
\hline$R_{1}$, w $R_{2}$ (all data) & $0.0526,0.0785$ & $0.0524,0.1086$ \\
\hline$C C D C$ & 1956795 & 1956797 \\
\hline
\end{tabular}

${ }^{\mathrm{a}} R_{1}=\sum|| F O|-| F C|| / \sum|F O|, \mathrm{w} R_{2}=\left\{\sum \mathrm{w}\left[(F O)^{2}-(F C)^{2}\right]^{2} / \sum \mathrm{w}\left[(F O)^{2}\right]^{2}\right\}^{1 / 2}$. 
Table S3. Summary of the crystal data and structure refinements for PLAPbBr and PLAPb.

\begin{tabular}{|c|c|c|}
\hline Compound name & PLAPbBr & PLAPb \\
\hline Empirical formula & $\mathrm{C}_{10} \mathrm{H}_{12} \mathrm{Br}_{2} \mathrm{O}_{6} \mathrm{~Pb}_{2}$ & $\mathrm{C}_{10} \mathrm{H}_{8} \mathrm{O}_{4} \mathrm{~Pb}$ \\
\hline Formula weight & 802.40 & 399.35 \\
\hline Crystal size $\left(\mathrm{mm}^{3}\right)$ & $0.2 \times 0.1 \times 0.1$ & $0.1 \times 0.1 \times 0.1$ \\
\hline Crystal system & triclinic & monoclinic \\
\hline Space group & $P-1$ & $P 2_{1} / c$ \\
\hline$T / \mathrm{K}$ & $292.41(16)$ & 150.01 \\
\hline$\lambda / \AA$ & 0.71073 & 0.71073 \\
\hline$a / \AA$ & 7.6163(7) & $8.5508(6)$ \\
\hline$b / \AA$ & $7.7759(7)$ & $13.1514(8)$ \\
\hline$c / \AA$ & $8.6569(7)$ & $8.5506(6)$ \\
\hline$\alpha /^{\circ}$ & $110.888(8)$ & 90 \\
\hline$\beta /^{\circ}$ & $95.650(7)$ & 91.64 \\
\hline$\gamma /{ }^{\circ}$ & $118.319(9)$ & 90 \\
\hline$V / \AA^{3}$ & $398.73(7)$ & $961.16(11)$ \\
\hline $\mathrm{Z}$ & 1 & 4 \\
\hline$D_{c} / \mathrm{g} \cdot \mathrm{cm}^{-3}$ & 3.342 & 2.760 \\
\hline$\mu / \mathrm{mm}^{-1}$ & 26.112 & 17.541 \\
\hline$F(000)$ & 354.0 & 728.0 \\
\hline $2 \theta$ for data collection $\left({ }^{\circ}\right)$ & $6.704-56.554$ & $4.766-52.838$ \\
\hline Measured refls. & 6321 & 32332 \\
\hline Independent refls. & 1980 & 1983 \\
\hline Observed reflection $(I>2 \sigma(I))$ & 2261 & 9781 \\
\hline Peak and hole/e $\AA^{-3}$ & $1.06 /-1.09$ & $0.77 /-0.78$ \\
\hline$R_{\text {int }}$ & 0.0436 & 0.0305 \\
\hline No. of parameters & 97 & 136 \\
\hline GOF & 1.002 & 1.140 \\
\hline${ }^{\mathrm{a}} R_{1}, \mathrm{w} R_{2}[I>2 \sigma(I)]$ & $0.0278,0.0470$ & $0.0124,0.0301$ \\
\hline$R_{1}$, w $R_{2}$ (all data) & $0.0380,0.0505$ & $0.0136,0.0307$ \\
\hline$C C D C$ & 1956796 & 1956798 \\
\hline
\end{tabular}

${ }^{\mathrm{a}} R_{1}=\sum|| F o|-| F c|| \Sigma|F o|, \mathrm{w} R_{2}=\left\{\sum \mathrm{w}\left[(F o)^{2}-(F c)^{2}\right]^{2} / \sum \mathrm{w}\left[(F o)^{2}\right]^{2}\right\}^{1 / 2}$. 


\section{Analysis of Short Ring-Interactions with Cg-Cg Distances < 6.0 Angstrom and Beta <}

\subsection{Deg.}

$$
\begin{array}{ll}
\mathrm{Cg}(\mathrm{I}) & =\text { Plane number } \mathrm{I}(=\text { ring number in }() \text { above }) \\
\alpha & =\text { Dihedral Angle between Planes I and } \mathrm{J}(\mathrm{Deg}) \\
\beta & =\text { Angle } \mathrm{Cg}(\mathrm{I}) \rightarrow \mathrm{Cg}(\mathrm{J}) \text { or } \mathrm{Cg}(\mathrm{i}) \rightarrow \text { Me vector and normal to plane I (Deg) } \\
\gamma & =\text { Angle } \mathrm{Cg}(\mathrm{I}) \rightarrow \mathrm{Cg}(\mathrm{J}) \text { vector and normal to plane } \mathrm{J}(\mathrm{Deg}) \\
\mathrm{Cg}-\mathrm{Cg} & =\text { Distance between ring Centroids (Ang.) } \\
\mathrm{CgI} \text { PPerp } & =\text { Perpendicular distance of } \mathrm{Cg}(\mathrm{I}) \text { on ring } \mathrm{J} \text { (Ang.) } \\
\text { CgJ_Perp } & =\text { Perpendicular distance of } \mathrm{Cg}(\mathrm{J}) \text { on ring I (Ang.) }
\end{array}
$$

Table S4. List of ring centroids about TPAPbBr.

\begin{tabular}{ll}
\hline $\mathrm{Cg}$ & atoms constituting the ring (plane) \\
\hline $\mathrm{Cg}(1)$ & $\mathrm{C}(2) \rightarrow \mathrm{C}(3) \rightarrow \mathrm{C}(4) \rightarrow \mathrm{C}(2) \rightarrow \mathrm{C}(3) \rightarrow \mathrm{C}(4) \rightarrow$ \\
$\mathrm{Cg}(2)$ & $\mathrm{C}(6) \rightarrow \mathrm{C}(7) \rightarrow \mathrm{C}(8) \rightarrow \mathrm{C}(11) \rightarrow \mathrm{C}(10) \rightarrow \mathrm{C}(9) \rightarrow$ \\
\hline
\end{tabular}

Table S5. List of short ring-interactions about TPAPbBr.

\begin{tabular}{lllllll}
\hline & Cg-Cg $(\AA)$ & $\alpha\left(^{\circ}\right)$ & $\beta\left(^{\circ}\right)$ & $\gamma\left(^{\circ}\right)$ & CgI_Perp $(\AA)$ & CgJ_Perp $(\AA)$ \\
$\mathrm{Cg}(\mathrm{I}) \rightarrow \mathrm{Cg}(\mathrm{J})^{1}$ & & & & & & \\
\hline $\mathrm{Cg}(1) \rightarrow \mathrm{Cg}(2)^{\mathrm{a}}$ & $3.631(6)$ & 4.73 & 23.48 & 19.17 & 3.429 & 3.330 \\
$\mathrm{Cg}(1) \rightarrow \mathrm{Cg}(2)^{\mathrm{b}}$ & $3.631(6)$ & 4.73 & 23.48 & 19.17 & 3.429 & 3.330 \\
$\mathrm{Cg}(2) \rightarrow \mathrm{Cg}(1)^{\mathrm{c}}$ & $3.631(6)$ & 4.73 & 19.17 & 23.48 & 3.330 & 3.429 \\
$\mathrm{Cg}(2) \rightarrow \mathrm{Cg}(1)^{\mathrm{d}}$ & $3.631(6)$ & 4.73 & 19.17 & 23.48 & 3.330 & 3.429 \\
$\mathrm{Cg}(2) \rightarrow \mathrm{Cg}(2)^{\mathrm{e}}$ & $5.370(6)$ & 0.03 & 58.83 & 58.83 & 3.169 & 3.169 \\
\hline
\end{tabular}

${ }^{1}$ Symmetry codes: (a) $1-X,-1 / 2+Y, 1 / 2-Z$; (b) $X, 1 / 2-Y, 1 / 2+Z$; (c) $1-X, 1 / 2+Y, 1 / 2-Z$; (d) $X, 1 / 2-Y,-1 / 2+Z$; (e) 1 $X,-Y,-Z$.

Table S6. List of ring centroids about TPAPb.

\begin{tabular}{ll}
\hline $\mathrm{Cg}$ & atoms constituting the ring (plane) \\
\hline $\mathrm{Cg}(3)$ & $\mathrm{C}(2) \rightarrow \mathrm{C}(3) \rightarrow \mathrm{C}(4) \rightarrow \mathrm{C}(5) \rightarrow \mathrm{C}(6) \rightarrow \mathrm{C}(7) \rightarrow$ \\
\hline
\end{tabular}

Table S7. List of short ring-interactions about TPAPb.

\begin{tabular}{lllllll}
\hline $\mathrm{Cg}(\mathrm{I}) \rightarrow \operatorname{Cg}(\mathrm{J})^{1}$ & $\mathrm{Cg}-\mathrm{Cg}(\AA)$ & $\alpha\left(^{\circ}\right)$ & $\beta\left(^{\circ}\right)$ & $\gamma\left(^{\circ}\right)$ & CgI_Perp $(\AA)$ & CgJ_Perp $(\AA)$ \\
\hline $\mathrm{Cg}(3) \rightarrow \operatorname{Cg}(3)^{\mathrm{a}}$ & $5.594(7)$ & 50.36 & 46.80 & 84.39 & 0.546 & 3.830 \\
$\mathrm{Cg}(3) \rightarrow \operatorname{Cg}(3)^{\mathrm{b}}$ & $3.981(6)$ & 14.68 & 25.87 & 33.20 & 3.332 & 3.582 \\
$\mathrm{Cg}(3) \rightarrow \operatorname{Cg}(3)^{\mathrm{c}}$ & $3.981(6)$ & 14.68 & 33.20 & 25.87 & 3.581 & 3.331 \\
$\mathrm{Cg}(3) \rightarrow \operatorname{Cg}(3)^{\mathrm{d}}$ & $5.478(7)$ & 52.75 & 44.93 & 84.78 & 0.499 & 3.877 \\
\hline
\end{tabular}

${ }^{1}$ Symmetry codes: (a) $1-X,-1 / 2+Y, 3 / 2-Z$; (b) $-1 / 2+X, Y, 3 / 2-Z$; (c) $1 / 2+X, Y, 3 / 2-Z$; (d) $1 / 2-X, 1 / 2+Y, Z$. 
Table S8. List of ring centroids about PLAPb.

\begin{tabular}{ll}
\hline $\mathrm{Cg}$ & atoms constituting the ring (plane) \\
\hline $\mathrm{Cg}(4)$ & $\mathrm{C}(3) \rightarrow \mathrm{C}(4) \rightarrow \mathrm{C}(5) \rightarrow \mathrm{C}(6) \rightarrow \mathrm{C}(7) \rightarrow \mathrm{C}(8) \rightarrow$ \\
\hline
\end{tabular}

Table S9. List of short ring-interactions about PLAPb.

\begin{tabular}{lllllll}
\hline $\mathrm{Cg}(\mathrm{I}) \rightarrow \mathrm{Cg}(\mathrm{J})^{1}$ & $\mathrm{Cg}-\mathrm{Cg}(\AA)$ & $\alpha\left(^{\circ}\right)$ & $\beta\left(^{\circ}\right)$ & $\gamma\left(^{\circ}\right)$ & CgI_Perp $(\AA)$ & CgJ_Perp $(\AA)$ \\
\hline $\mathrm{Cg}(4) \rightarrow \mathrm{Cg}(4)^{\mathrm{a}}$ & $4.4370(15)$ & 52.21 & 11.16 & 41.78 & 3.309 & 4.353 \\
$\mathrm{Cg}(4) \rightarrow \mathrm{Cg}(4)^{\mathrm{b}}$ & $4.4369(15)$ & 52.21 & 41.78 & 11.16 & 4.353 & 3.309 \\
\hline
\end{tabular}

${ }^{1}$ Symmetry codes: (a) $X, 3 / 2-Y,-1 / 2+Z$; (b) $X, 3 / 2-Y, 1 / 2+Z$.

Table S10. A summary of thermal stability and PL properties of the title compounds and other $\mathrm{Pb}$-based $\mathrm{COMH}$.

\begin{tabular}{|c|c|c|c|c|c|c|}
\hline $\begin{array}{l}\text { Properties } \\
\text { Compounds }\end{array}$ & Life Time & QYs (\%) & TG $\left({ }^{\circ} \mathrm{C}\right)$ & $\lambda_{\text {ex }}(\mathrm{nm})$ & $\lambda_{\mathrm{em}}(\mathrm{nm})$ & Ref \\
\hline $\mathrm{Pb}_{4} \mathrm{Br}_{2}(\mathrm{TPA})_{3}$ & $1.035 \mathrm{~ms}(293 \mathrm{~K})$ & 25.98 & 420 & 330 & 505 & This work \\
\hline TPA & $2.86 \mathrm{~ms}(293 \mathrm{~K})$ & 0.5 & - & & 382 & {$[3]$} \\
\hline $\mathbf{P b}_{2} \mathbf{B r}_{2}(\mathbf{P L A})$ & $53.3 \mathrm{~ms}(293 \mathrm{~K})$ & 6.05 & 45 & 370 & 450 & This work \\
\hline $\mathbf{P b}_{2} \mathbf{B r}_{2}$ (APA) & $2.65 \mathrm{~ns}(293 \mathrm{~K})$ & 2.0 & 200 & 356 & 565 & \multirow{2}{*}{ [4] } \\
\hline $\mathbf{P b}_{2} \mathrm{Cl}_{2}$ (APA) & $2.24 \mathrm{~ns}(293 \mathrm{~K})$ & 11.8 & 200 & 327 & 536 & \\
\hline $\mathrm{PbBr}_{2}(\mathrm{bp4mo})$ & $250 \mu \mathrm{s}$ & 22 & 250 & 380 & 600 & \multirow{2}{*}[5]{} \\
\hline $\mathrm{PbCl}_{2}(\mathrm{bp} 4 \mathrm{mo})$ & $250 \mu \mathrm{s}$ & 6 & 200 & 380 & 600 & \\
\hline $\mathbf{P b}_{2} \mathbf{B r}_{2}(\mathbf{A P A})$ & $1.7 \mathrm{~ns}(293 \mathrm{~K})$ & 5.6 & 250 & 360 & 530 & \multirow[t]{2}{*}{ [6] } \\
\hline $\mathbf{P b}_{3} \mathrm{Br}_{4}(\mathrm{SCA})$ & $1.6 \mathrm{~ns}(293 \mathrm{~K})$ & 1.8 & 250 & 370 & 480 & \\
\hline $\begin{array}{l}\mathrm{Pb}_{2} \mathrm{Br}_{3}(\mathrm{TPA})_{2} \\
\quad(\mathrm{DMF})_{3}\end{array}$ & 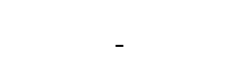 & - & 175 & 270 & 514 & [7] \\
\hline $\begin{array}{c}\mathrm{Pb}_{2} \mathrm{Br}_{3}(\mathrm{TPA})_{2} \\
(\mathrm{DMF})_{3}\end{array}$ & $0.53 \mu \mathrm{s}(77 \mathrm{~K})$ & 1.5 & 180 & 380 & 561 & \multirow{2}{*}[8]{} \\
\hline $\begin{array}{l}\mathrm{Pb}_{2} \mathrm{Cl}_{3}(\mathrm{TPA})_{2} \\
\quad(\mathrm{DMF})_{3}\end{array}$ & $1.72 \mathrm{~ns}(293 \mathrm{~K})$ & $6-8$ & 180 & 367 & 560 & \\
\hline $\mathrm{Pb}_{3} \mathrm{Br}(\mathrm{bpdc})_{2}$ & $53.9 \mathrm{~ns}(77 \mathrm{~K})$ & - & - & 300 & 402 & {$[8]$} \\
\hline $\mathrm{Pb}_{2} \mathrm{Br}_{2}(\mathrm{CHDA})$ & $96.7 \mathrm{~ns}(239 \mathrm{~K})$ & 17.2 & 330 & 345 & 500 & \multirow{2}{*}{ [9] } \\
\hline $\mathrm{Pb}_{2} \mathrm{Cl}_{2}(\mathrm{CHDA})$ & $215 \mu \mathrm{s}$ & 20 & 260 & 304 & 433 & \\
\hline
\end{tabular}

APA= Adipic acid;bp4mo=4,4'-bipyridine N-oxide (bp4mo); SCA=Succinic acid; bpdc=4,4'-Biphenyldicarboxylic acid;

CHDA=trans-1,4-cyclohexanedicarboxylic acid.

There are two B alert in the Checkcif report.

For TPAPb crystal:

PLAT972_ALERT_2_B Check Calcd Resid. Dens. 0.72A From Pb1 -2.68 eA-3.

Author Response: The alert usually arises from the unassigned q peak. We have recollected the SCXRD data of TPAPb several times. However, the data quality is still not ideal. The electron density is around the heavy $\mathrm{Pb}$ centres. Thus, such residual density is likely not from unaccounted atom types. 
For PLAPb crystal:

LAT430_ALERT_2_B Short Inter D...A Contact O3 ..O3 2.76 Ang.

Author Response: The ligand carboxylate groups are close to neighboring carboxylate groups.

\section{Reference}

[1] G. Sheldrick, Acta Crystallogr. Sect. C 2015, 71, 3-8.

[2] O. V. Dolomanov, L. J. Bourhis, R. J. Gildea, J. A. K. Howard, H. Puschmann, J. Appl. Crystallogr. 2009, 42, 339-341.

[3] X. Yang, D. Yan, Adv. Opt. Mater. 2016, 4, 897-905.

[4] Z. Zhuang, C. Peng, G. Zhang, H. Yang, J. Yin, H. Fei, Angew. Chem. Int. Ed. 2017, $56,14411-14416$.

[5] O. Toma, N. Mercier, M. Allain, F. Meinardi, C. Botta, Eur. J. Inorg. Chem. 2017, 2017, 844-850.

[6] C. Peng, Z. Zhuang, H. Yang, G. Zhang, H. Fei, Chem. Sci. 2018, 9, 1627-1633.

[7] S. X. Liu, G. J. Yuan, L. Zhai, L. Wang, H. B. Luo, X. M. Ren, Dalton. Trans. 2018, 47, 14233-14240.

[8] C. Peng, X. Song, J. Yin, G. Zhang, H. Fei, Angew. Chem. Int. Ed. 2019, 56, 1441114416.

[9] J. Yin, H. Yang, H. Fei, Chem. Mater. 2019, 31, 3909-3916. 